\title{
Il patrimonio fotografico dell'Archivio Storico delle Gallerie Fiorentine: ragioni e provenienza di alcune fotografie dello stabilimento Brogi
}

\begin{abstract}
This essay considers a selection of painting reproductions made by Brogi in the 1870s, as part of a larger study on the historical archive of the Florentine Galleries held by the Superintendence of Archaeology, Fine Arts and Landscape in Florence. Cross-referencing these photographs with written documents in the same archive and the commercial catalogs published by Brogi between 1863 and 1901, it is possible to determine that they were originally delivered in compliance with legal deposit regulations established by the new Ministry of Public Education in 1867. At the same time, this case study sheds new light on the connections between commercial photographers and art institutions in Italy in the second half of the $19^{\text {th }}$ century, especially regarding the creation of public photographic archives and the role played by Corrado Ricci, the director of the Uffizi Galleries between 1903 and 1906.
\end{abstract}

\section{Keywords}

FLORENCE; GALLERIE DEGLI UFFIZI; BROGI COMPANY;

PHOTOGRAPHIC REPRODUCTIONS; PHOTOGRAPHIC ARCHIVES;

HISTORICAL ARCHIVE UFFIZI; SANZIO, RAFFAELLO; RICCI, CORRADO

Q

uesto studio prende in esame alcune fotografie della ditta Brogi rinvenute nell'Archivio Storico delle Gallerie Fiorentine e le pone in relazione con la documentazione manoscritta presente nel medesimo archivio, in particolare per quanto riguarda i permessi d'accesso alle gallerie richiesti dai fotografi e il lascito della doppia copia per ogni soggetto riprodotto ${ }^{-1}$. L'analisi della documentazione manoscritta e delle fotografie conduce in due direzioni di ricerca parallele: una più microscopica, relativa alla produzione fotografica di 
Brogi; l'altra, di più ampio respiro, riguardante la connessione di queste fotografie con quello che fu l'Archivio fotografico degli Uffizi, voluto dal direttore Corrado Ricci nel 1903. Le due tematiche - fattura e provenienza delle fotografie presenti nell'Archivio Storico delle Gallerie sono trattate nel testo che segue con una prospettiva che va dal micro al macro, presentando i primi risultati di una ricerca ancora in corso. Iniziamo dal dato che motiva la presenza delle fotografie di Brogi alle Gallerie e poi in archivio. Si tratta del decreto emanato dal Ministero della Pubblica Istruzione il 14 ottobre 1867 che aveva stabilito, per tutto il territorio nazionale, l'obbligo di consegna di due stampe fotografiche per ogni negativo tratto da oggetti appartenenti alle collezioni pubbliche, da depositare rispettivamente una alla sede locale museale ove si conservava l'opera e l'altra al Ministero. Regolamento che imponeva anche una serie di comportamenti, fra i quali: "3. Che in nessun caso e per nessun motivo sia concesso di rimuovere dal posto, ov’è collocato, l'oggetto che si vuol riprodurre. 4. Che di ogni fotografia una copia si dia alla Galleria, ed un'altra a questo Ministero, 5. Che sia vietato di far traffico delle fotografie entro il locale della Galleria" -2

Il tema della doppia copia s'impone di prim'ordine nel momento in cui si decide di affrontare, nonché storicizzare, le attività dei fotografi nei musei: non soltanto per la questione in sé, su cui si innesta il sistema che ha permesso ai fotografi di costituire i propri repertori commerciali legati alle riproduzioni di opere d'arte mobili ${ }^{-3}$, ma anche perché questi lasciti formarono, secondo dinamiche differenti, una porzione degli archivi fotografici del sistema centrale di tutela e di quello periferico delle soprintendenze ${ }^{-4}$.

Le potenzialità di questa indagine si sono manifestate nel corso di una ricerca più ampia sui fotografi a Firenze nel secondo Ottocento. La scarsità di fonti primarie e secondarie mi ha spinto alla ricerca di eventuali tracce nei luoghi in cui i fotografi hanno operato, primi fra tutti i musei. Lo spoglio sistematico dei manoscritti conservati nell'Archivio storico delle Gallerie Fiorentine, che integra i documenti emersi dalle ricerche di Chiara Migliorini e Monica Maffioli sull'Accademia di Belle Arti e sull'Accademia delle arti del disegno entrambi presenti in città, discende quindi dalla necessità di colmare anzitutto una lacuna d'informazioni storiche ${ }^{-5}$.

\section{Le fotografie depositate per obbligo nei musei}

La ricognizione nell'Archivio Storico delle Gallerie Fiorentine, sull'arco cronologico 1855-1890, ha restituito una vasta documentazione delle attività fotografiche nei musei della città che consiste soprattutto nelle richieste di permesso per fotografare il patrimonio artistico e che implica questioni di carattere burocratico, diplomatico, etico, tecnico-pratico e legale. Posta in relazione con l'ambito di produzione dei fotografi, questa documentazione delinea un vero e proprio sistema di rapporti e pratiche tra gli ateliers commerciali e gli organi di tutela - in special modo le direzioni dei musei e il Ministero della Pubblica Istruzione - in 
una continua ricerca di equilibrio tra esigenze degli attori privati e obblighi di quelli pubblici ${ }^{-6}$.

L'assidua insistenza da parte della Direzione delle Gallerie e del Ministero della Pubblica Istruzione affinché fosse rispettato il vincolo posto a ogni richiesta di autorizzazione a fotografare, che emerge chiaramente dalla consistenza archivistica, denota l'importanza del lascito per le istituzioni ${ }^{-7}$. Tuttavia, nelle lettere non si menziona mai il destino delle fotografie depositate, forse perché la loro finalità non era stata stabilita a priori sia dagli uffici locali sia da quelli ministeriali. Un secondo aspetto che emerge con evidenza è la prova documentaria dei ripetuti lasciti della ditta Brogi, che peraltro trovano concordanza cronologica con la pubblicazione dei cataloghi commerciali della ditta stessa. Un terzo riscontro, infine, è dato dallo studio di alcune stampe presenti nell'Archivio Storico delle Gallerie Fiorentine, la cui analisi, come vedremo a breve, mi ha condotto a identificarle con le fotografie depositate dai fotografi Brogi negli anni Settanta dell'Ottocento.

Quest'ultima questione merita di essere approfondita prima di entrare nel vivo della ricerca sul caso Brogi. Negli studi finora condotti non si hanno notizie precise sulla conservazione delle fotografie lasciate dai fotografi negli archivi dei musei fiorentini; pertanto, per individuarne la possibile presenza, dopo aver appurato che non erano state conservate nel Gabinetto Fotografico di Soprintendenza (fondato nel 1904) ${ }^{-8}$, si è constatato che l'Archivio Storico restava l'unico luogo possibile degli Uffizi dove cercarle, poiché la destinazione d'uso più plausibile delle riproduzioni fotografiche delle opere dei musei pareva essere quella di supporto iconografico alla catalogazione e alla conservazione del patrimonio museale. In effetti, nei dossier conservati nello schedario dell'Archivio Storico in ordine alfabetico per autore, vi sono notizie storico-critiche sulle singole opere d'arte conservate nelle Gallerie (che iniziano delle note manoscritte ottocentesche per arrivare fino alle schede OA dattiloscritte degli anni Novanta del secolo scorso), e una piccola e non omogenea parte di fotografie. Molte di queste sono riconducibili alle campagne fotografiche degli stabilimenti fotografici delle quali si trova riscontro documentario nell'Archivio Storico. Complessivamente, si tratta di corpus iconografico costituito da stampe fotografiche di varie epoche, tecniche e produzioni, dalle più antiche albumine di Alinari, Brogi, Jacquier e Mannelli a fotografie della seconda metà del Novecento a opera della stessa Soprintendenza ${ }^{-9}$.

\section{Proposta di metodo: il gioco delle tre fonti}

La ricerca si è articolata partendo dall'analisi di fonti differenti, documentali e fotografiche, in cui la corrispondenza tra il documento manoscritto e la fotografia ha trovato un primo riscontro nel catalogo commerciale del fotografo, cartina di tornasole (ma anche fonte insidiosa) di quell'attività di traduzione del patrimonio artistico operato dai fotografi attraverso le campagne fotografiche fra Ottocento e Novecento. 
A cominciare dal documento d'archivio, prodotto dalla Direzione delle Gallerie o dal Ministero della Pubblica Istruzione in merito all'autorizzazione rilasciata ed eventualmente al deposito di fotografie ricevute secondo il regolamento ministeriale, la ricerca è proseguita con la verifica della presenza delle stesse in archivio e il contestuale riscontro nel catalogo di vendita del fotografo. Di seguito daremo un esempio di come è stato possibile far dialogare correttamente le tre fonti attraverso il caso di studio cui si è accennato poc'anzi: l'attività dei fotografi Brogi nelle Gallerie Fiorentine.

\section{L'attività dei fotografi Brogi nelle Gallerie Fiorentine (1875-1881)}

La ditta Brogi, attiva a Firenze col capostipite Giacomo dal 1856, divenne presto protagonista nel panorama della fotografia italiana ed europea ${ }^{-10}$. Specializzata nel ritratto, la ditta Brogi (attiva fino al 1950) divenne negli anni Settanta dell'Ottocento un atelier di punta ampliando l'offerta commerciale con vedutismo e nella riproduzione del patrimonio artistico con tre punti vendita a Firenze, Roma e Napoli. Nel 1881, la ditta passò al figlio di Giacomo, Carlo, che si dedicò anche alla lotta per la tutela dei diritti fotografici pubblicando anche testi sul tema e fu, inoltre, tra i promotori della Società Fotografica Italiana fondata a Firenze nel 1889. A oggi sono stati rinvenuti 19 cataloghi di vendita che attestano la sua produzione, editi tra il 1865 e il 1932. Se è datato al 1863 il primo catalogo commerciale $-{ }^{11}$, ma che non risulta rintracciabile, il primo disponibile alla consultazione, e fino a oggi inedito, risale al 1865, con il titolo Catalogo della Pinacoteca Universale, grandiosa raccolta fotografica d'insigni dipinture disegni ecc. ${ }^{-12}$, in cui l'aggettivo "universale" dichiara quella 'vocazione enciclopedica' considerata peculiare anche per la produzione coeva Alinari ${ }^{-13}$ assieme alla presenza di ben 1088 soggetti. Probabilmente, almeno per quanto riguarda i soggetti conservati all'estero, si tratta di fotografie eseguite da altri fotografi: se prendiamo l'esempio del Louvre, gli studi testimoniano che negli anni Sessanta gli ingressi dei fotografi erano limitati a quei pochi già ritenuti esperti, come Charles Marville. Inoltre, l'analisi dei documenti degli Archives des Musées Nationaux, fatta da Laure Boyer, non ha rilevato l'ingresso di fotografi italiani in quest'epoca. A rafforzare l'idea che queste prime fotografie dei soggetti esteri non fossero di mano di Brogi, si aggiunge il fatto che egli avesse iniziato la sua attività negli anni Cinquanta proprio vendendo fotografie realizzate da altri ${ }^{-14}$.

Dopo questa edizione bisognerà attendere il 1874 per un nuovo catalogo, nel quale il fotografo si concentra principalmente su soggetti fiorentini ${ }^{-15}$. Il frontespizio dichiara che le fotografie sono tratte dagli originali: si tratta di 498 soggetti tratti dall'Accademia di Belle Arti di Firenze, dai disegni e i dipinti degli Uffizi, dagli affreschi del Chiostro dello Scalzo, dal Palazzo Medici Riccardi, dal Campo Santo di Pisa, dalla Regia Pinacoteca di Torino, dalla Galleria Moderna di Palazzo Pitti e, per concludere, un'appendice di soggetti ritraenti piante e frutta. Per quanto riguarda i soggetti dell'Accademia, accenniamo brevemente alla 
documentazione presente nell'Archivio Storico dell'Accademia di Belle Arti di Firenze, luogo in cui Brogi riversa l'intera collezione di soggetti riprodotti, ben 404 fotografie: "Con la lettera si allega n. 332 fotografie dei quadri da me riprodotti le quali unite alle 72 rimesse già il 27 marzo formano il totale della mia collezione in cagione di due copie per ogni soggetto" ${ }^{-16}$. Il catalogo del 1874 è seguito da altri quattro cataloghi (tre nel 1876 e uno nel 1878) che, come vedremo, concordano cronologicamente coi lasciti delle doppie copie al museo. Tuttavia la ricerca su Brogi si estende fino al 1881: sono questi, infatti, gli anni in cui Giacomo incrementa la propria attività, lasciando traccia nelle carte dell'archivio storico delle Gallerie Fiorentine con almeno quindici fascicoli che ben si prestano a fare da campione per la nostra indagine. Tali pratiche riguardano sia richieste di permesso per fotografare il patrimonio artistico, sia attestazioni del deposito della doppia copia obbligatorio per regolamento dal 1867.

La prima pratica rintracciata in archivio risale al 1875 e tratta della richiesta di permesso per fotografare nelle Gallerie e in San Marco ${ }^{-17}$. Tale produzione coincide con i due cataloghi monografici editi da Brogi l'anno seguente, dedicati rispettivamente agli Uffizi e al Museo di San Marco ${ }^{-18}$.

Nel febbraio del 1877 Brogi dichiara di aver inviato agli Uffizi, "in conformità con le direttive ministeriali”, due copie di ciascun negativo eseguito nella galleria ${ }^{-19}$.

Tuttavia, nei documenti relativi alle pratiche del 1875 e del 1877 non sono resi noti i soggetti che il fotografo aveva intenzione di ritrarre, fatto che non consente di compiere controlli mirati sul patrimonio fotografico a disposizione nell'Archivio Storico. È invece l'operazione successiva nelle Gallerie, quella del 1878 , a fornirci un caso adeguato a testare il metodo d'incrocio delle tre fonti: documento d'archivio, fotografia, catalogo del fotografo. Si tratta dell'autorizzazione a fotografare nella Galleria Palatina per la quale Brogi aveva specificato un breve elenco di soggetti:

Vista la domanda del Sig. Brogi di poter riprodurre in fotografia i qua-
dri: la Madonna della Seggiola, la Madonna del Granduca, la Madonna
del Baldacchino, la Madonna del Morillo, la Madonna di Tiziano, la
Bella di Tiziano, il Concerto di Giorgione, io le do facoltà di concedere
al Brogi quel permesso; il quale dovrà incominciare ad avere effetto
dopo che l'Alinari avrà finito la stessa operazione, ed avvertendo di
non rimuovere i quadri o almeno quelli di maggior pregio. Ella abbia
ancora la compiacenza di avvisare quel fotografo che egli deve di tutte
quelle copie fotografate concedere due copie, una pel Ministero ed
una per le Gallerie. II Ministro -20

Nel medesimo anno - siamo nel 1878 - è pubblicato un nuovo catalogo di vendita, in cui le opere di Palazzo Pitti rappresentano una novità 
tra i soggetti offerti dalla ditta ${ }^{-21}$. Inoltre, nel marzo del 1879, Brogi effettua un versamento di 101 fotografie al museo:

La Direzione scrivente accusa ricevimento alle SS. LL. delle 53 fotografie testé spedite e delle altre 48 inviate precedentemente, giusta la disposizione del Regolamento approvato dal Ministero intorno alle riproduzioni di capi d'arte ${ }^{-22}$.

Lo stesso giorno, il 20 marzo del 1879, il direttore delle Gallerie scrive al Ministro avvisandolo dell'invio di 70 fotografie, realizzate da diversi fotografi che lo scrivente aveva richiamato all'ordine per non aver depositato la doppia copia dopo aver realizzato negativi da oggetti delle collezioni fiorentine. Una traccia importante questa che testimonia la circolazione di fotografie d'arte tra i tre interlocutori, fotografo, museo e Ministero: "La maggior parte di dette fotografie furono ritirate da me con invito speciale ai fotografi che non avevano soddisfatto ancora l'obbligo loro. Altre esistevano già eppur esse in doppio esemplare nell'Archivio di questa direzione" ${ }^{23}$.

La pubblicazione del nuovo catalogo di vendita determina quindi la data d'ingresso delle rispettive fotografie in archivio. Ma andiamo per ordine. Effettivamente, dal controllo svolto sulla documentazione fotografica dell'Archivio Storico, sono emerse 4 fotografie dei sette soggetti elencati nella lettera sopra citata: si tratta di stampe all'albumina su carta sottile di medio formato, i cui numeri nella didascalia editoriale corrispondono al numero d'ordine nel catalogo ${ }^{-24}$. La Madonna della Seggiola corrisponde nel catalogo al numero d'ordine 2905 (fig. 1): la fotografia conservata alle Gallerie è montata su un supporto in carta e quindi non sono visibili eventuali tracce sul verso. Invece sul supporto compare, tra varie altre note manoscritte, il timbro a inchiostro "Ufficio Ricerche della Soprintendenza Archeologica, Belle arti e Paesaggio di Firenze" ${ }^{25}$.

La Madonna del Granduca compare anch'essa per la prima volta nel catalogo del 1878, come conferma il numero 2832 nella didascalia editoriale, analogo a quello d'ordine nel catalogo. Sul verso della fotografia (fig. 2) compaiono due timbri a inchiostro "Ufficio Ricerche" e "Archivio delle RR. Gallerie di Firenze".

Stessa lettura per la Madonna del Baldacchino (numero di catalogo 2837) e per il Concerto di Tiziano, all'epoca ancora attribuito a Giorgione (2836). Della Bella di Tiziano non è presente in archivio la riproduzione dell'intero dipinto inserito in catalogo nel 1878, ma un particolare della testa che entrerà in catalogo successivamente e che risulta ancora presente in quello del $1903\left(2948^{\mathrm{a}}\right)$, mentre non sono state rintracciate né la Madonna di Tiziano né la Madonna del Murillo.

Nel caso specifico emerge quindi, come inizialmente supposto, la consonanza tra le diverse fonti esaminate, a dimostrazione del sistema di relazioni che s'instaurò in quegli anni tra i musei e i fotografi. Stando alla documentazione dell'Archivio Storico, l'attività dei Brogi riprende 


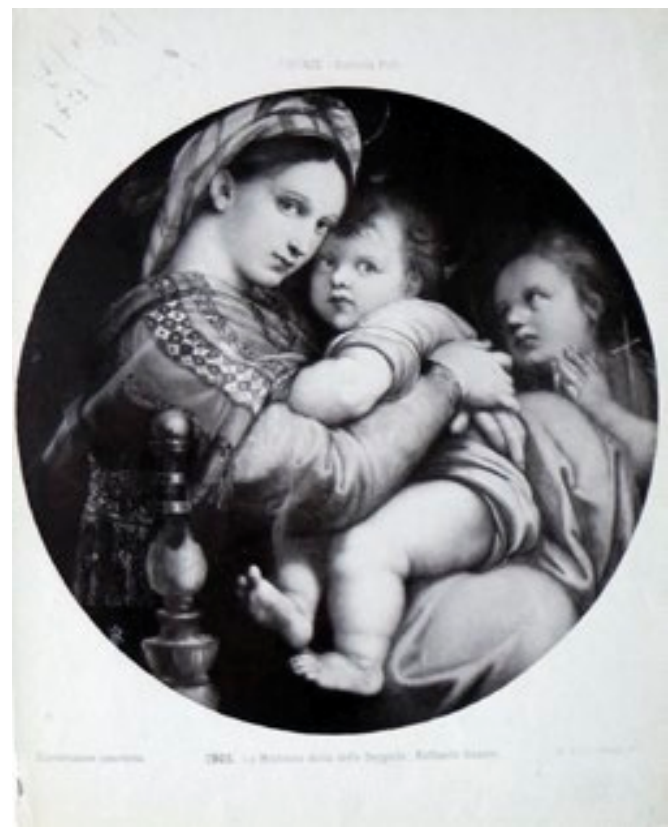

01

\section{Brogi,}

Raffaello: dettaglio della Madonna della Seggiola, 1878.

Stampa all'albumina,

$24,5 \times 19,2 \mathrm{~cm}$.

Firenze, ASGF, dossier

"Raffaello Inv. Palatina”.

Riproduzione su

concessione del Ministero

per i Beni e le Attività

Culturali

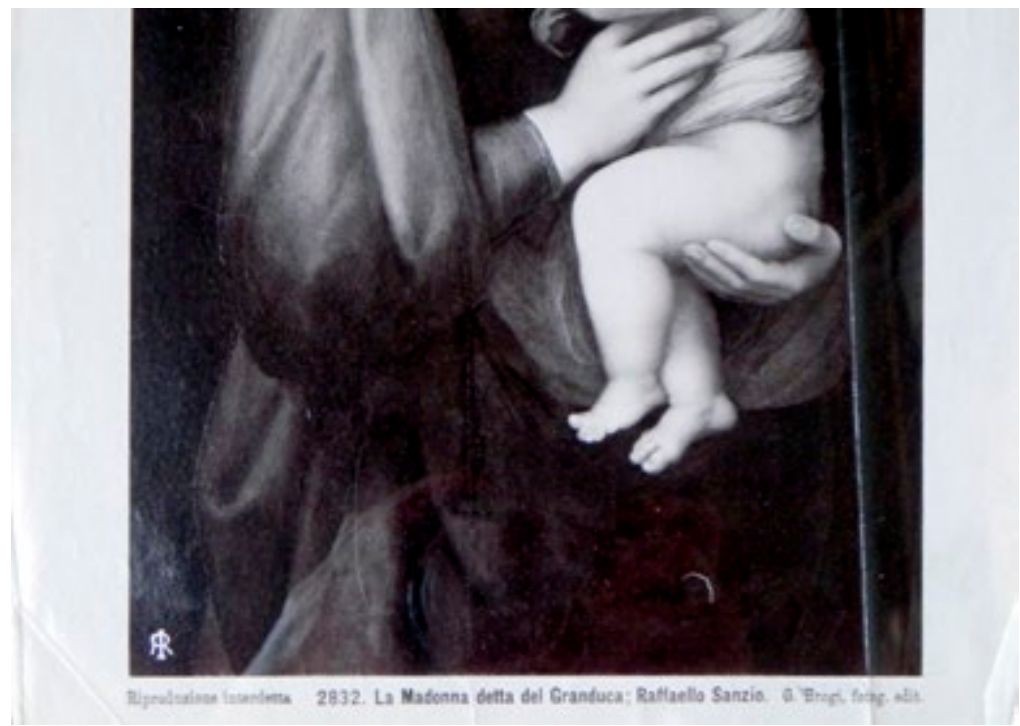

\section{Brogi,}

Raffaello: Madonna del Granduca, 1878.

Stampa all'albumina, $25 \times 19,4 \mathrm{~cm}$, dettaglio didascalia.

Firenze, ASGF, dossier "Raffaello Inv. Palatina". Riproduzione su concessione del Ministero per i Beni e le Attività Culturali 
nell'estate del 1880 con due richieste corredate di note sui soggetti da ritrarre. Questa pratica è composta da sei lettere manoscritte e un telegramma inviato da Brogi al Ministero della Pubblica Istruzione con la richiesta trascritta: concerne due istanze di permesso per dipinti e statue delle gallerie, la prima di luglio in cui si elencano cinque opere di Rubens e una di Bachiacca, e la seconda di agosto in cui si dichiara una lista di tredici dipinti di antichi maestri e capolavori quali quelli di Botticelli, Andrea del Sarto, Mantegna e Leonardo da Vinci ${ }^{-26}$. L'anno seguente, presenta istanza per fotografare otto opere di cui deposita subito le riproduzioni. Nel febbraio del 1881 i fotografi Brogi richiedono di poter fotografare tre soggetti: il Crocifisso affrescato da Perugino nel refettorio dell'ex convento di Santa Maria Maddalena; un altro Crocifisso affrescato da Beato Angelico in San Marco e il San Sebastiano del Sodoma esposto agli Uffizi ${ }^{-27}$. Per tutto il decennio i rapporti tra i fotografi Brogi e le Gallerie Fiorentine proseguiranno ${ }^{-28}$.

\section{Gli oggetti fotografici dell'Archivio Storico delle Gallerie, indicatori di un patrimonio più vasto: il Regio Archivio Fotografico degli Uffizi}

Si potrebbero dunque condurre altre verifiche sull'attività Brogi, poiché la documentazione è ricca di spunti. Tuttavia a questo punto ritengo più opportuno focalizzare l'attenzione sul patrimonio fotografico presente nell'Archivio Storico per approfondirne l'analisi materiale e la sua valenza storica, poiché è la prima volta che tali documenti vengono esaminati nel loro contesto sedimentale. Ho concentrato l'attenzione sulle albumine su carta sottile, la tecnica di stampa più diffusa dall'età del collodio. Cosciente del potenziale epistemologico degli oggetti fotografici ${ }^{29}$, nelle fotografie ho cercato di individuare, oltre alle tecniche e ai supporti, anche le tracce indicative di determinati ambiti di produzione e d'uso. Sono emersi numerosi indizi riscontrabili sul verso della fotografia, spesso indice di impieghi e destinazioni che l'oggetto fotografico ha subito nel tempo. La presenza di timbri è frequente: alcuni confermano l'appartenenza della fotografia alle Gallerie, altri denotano una diversa provenienza, ad esempio da un archivio privato quale la fototeca del conte Carlo Gamba ${ }^{\mathbf{3 0}}$, oppure l'archivio Filippo Rossi, pertinente all'omonimo funzionario ${ }^{-31}$. Vi sono fotografie di produzione privata come quelle degli stabilimenti fotografici Alinari, Brogi, Jacquier, Mannelli, oppure realizzate dalla stessa Soprintendenza, in un arco cronologico che va dagli anni Settanta dell'Ottocento fino a tutto il Novecento. Si profila così un patrimonio diversificato, per tipologia e provenienza, che di seguito analizzerò attraverso un campione scelto allo scopo di rispondere al quesito già posto da Costanza Caraffa - "che cosa documentano effettivamente queste fotografie?" - 32 - a cui potremmo aggiungere: che cosa documentano oggi?.

È necessario fare un passo indietro e riprendere un tema fin qui solo accennato e relativo al fatto che le fotografie depositate per obbligo dai fotografi hanno contribuito almeno in parte alla sedimentazione di quei patrimoni fotografici che oggi costituiscono gli archivi di documentazione 
iconografica dei musei e dei gabinetti fotografici delle Soprintendenze. Nelle fotografie rintracciate nell'Archivio storico delle Gallerie Fiorentine, plurime sono le tracce presenti sul verso, attestanti i vari passaggi amministrativi e le sedi conservative all'interno delle Gallerie, ad esempio quelli relativi a "G. Firenze", "R. Galleria di Fir.", "Archivio delle R. R. Gallerie di Firenze”, "R. Sopraintendenza all'arte medioevale e moderna per la Toscana (Ufficio Catalogo) Firenze", "Soprintendenza per i beni artistici e storici - Firenze - Ufficio Ricerche" - ${ }^{33}$.

Oltre ai timbri, che possono aiutare a individuare una più esatta cronologia delle stampe fotografiche sulla base delle denominazioni di musei e uffici, la traccia che più colpisce è la nota manoscritta a inchiostro di un numero, talvolta seguito dal nome del fotografo, dall'indicazione dell'artista e del titolo dell'opera d'arte quando l'immagine è sprovvista di didascalia. Il numero manoscritto, nei casi qui contemplati, non corrisponde né al numero d'inventario del dipinto all'interno del museo, né al numero d'ordine del catalogo commerciale del fotografo. Pertanto ho deciso di approfondire la questione analizzando un campione selezionato delle fotografie Brogi: si tratta del gruppo di soggetti raffaelleschi della Galleria Palatina, di cui fa parte anche la fotografia della Madonna del Granduca di cui si è già parlato (fig. 2). La scelta di Raffaello sta nell'ordine della quantità di opere del maestro presenti nelle Gallerie e dunque nella relativa documentazione catalografica tale da costituire un valido campione d'indagine. I faldoni di Raffaello consultati nello schedario sono due: "Raffaello Palatina Ritratti" che comprende i dossier di 7 opere con all'interno 7 fotografie di Brogi e "Raffaello Inv. Palatina" che comprende i dossier di 7 opere in cui si conservano 11 fotografie Brogi. In questo secondo faldone si trova anche il dossier de La Muta, in realtà esposta all'epoca agli Uffizi.

Presenteremo le fotografie, inserite nel fascicolo intitolato "Inv. Raffaello Palatina" conservato nello schedario dell'Archivio Storico delle Gallerie Fiorentine, descrivendo prima il verso e seguendo l'ordine progressivo del numero manoscritto a inchiostro sul margine basso. Si tratta di 7 stampe all'albumina che per dimensione, qualità della carta e della stampa, caratteristiche tipografiche delle didascalie editoriali e per il numero d'ordine del soggetto nel catalogo commerciale appartengono alla produzione Brogi degli anni Settanta e Ottanta ${ }^{34}$.

Il verso della prima fotografia (fig. 3) porta il numero manoscritto "1044", due timbri a inchiostro - quello della "Soprintendenza ai Beni Artistici” e quello dell'“Archivio delle Regie Gallerie” (posto in una doppia cornice circolare) - nonché l'annotazione manoscritta "Palatina n. 94". Si tratta di un dettaglio del dipinto della Madonna dell'Impannata, coi volti della Vergine e del bambino, che secondo il numero "2940a", riportato nella didascalia editoriale, entra nel catalogo Brogi dopo il 1878 ed è ancora presente nel $1903^{-35}$.

Il verso della seconda fotografia (fig. 4) reca il numero manoscritto "1045" e il timbro dell'“Archivio delle Regie Gallerie”: si tratta del dettaglio del ritratto del San Giovannino nella Madonna dell'Impannata 


\section{Brogi,}

Raffaello: Madonna dell'Impannata, 1878.

Stampa all'albumina, $25,1 \times 19,4 \mathrm{~cm}$, dettaglio del verso.

Firenze, ASGF, dossier "Raffaello Inv. Palatina". Riproduzione su concessione del Ministero per i Beni e le Attività Culturali
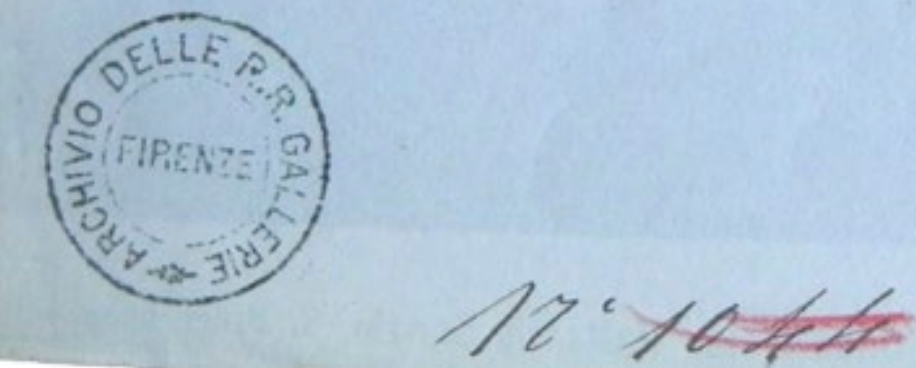

\section{4}

\section{Brogi,}

Raffaello: Madonna dell'Impannata dettaglio di San Giovannino, 1878.

Stampa all'albumina, $25,2 \times 19,5 \mathrm{~cm}$, dettaglio del verso.

Firenze, ASGF, dossier "Raffaello Inv. Palatina". Riproduzione su concessione del Ministero per i Beni e le Attività Culturali

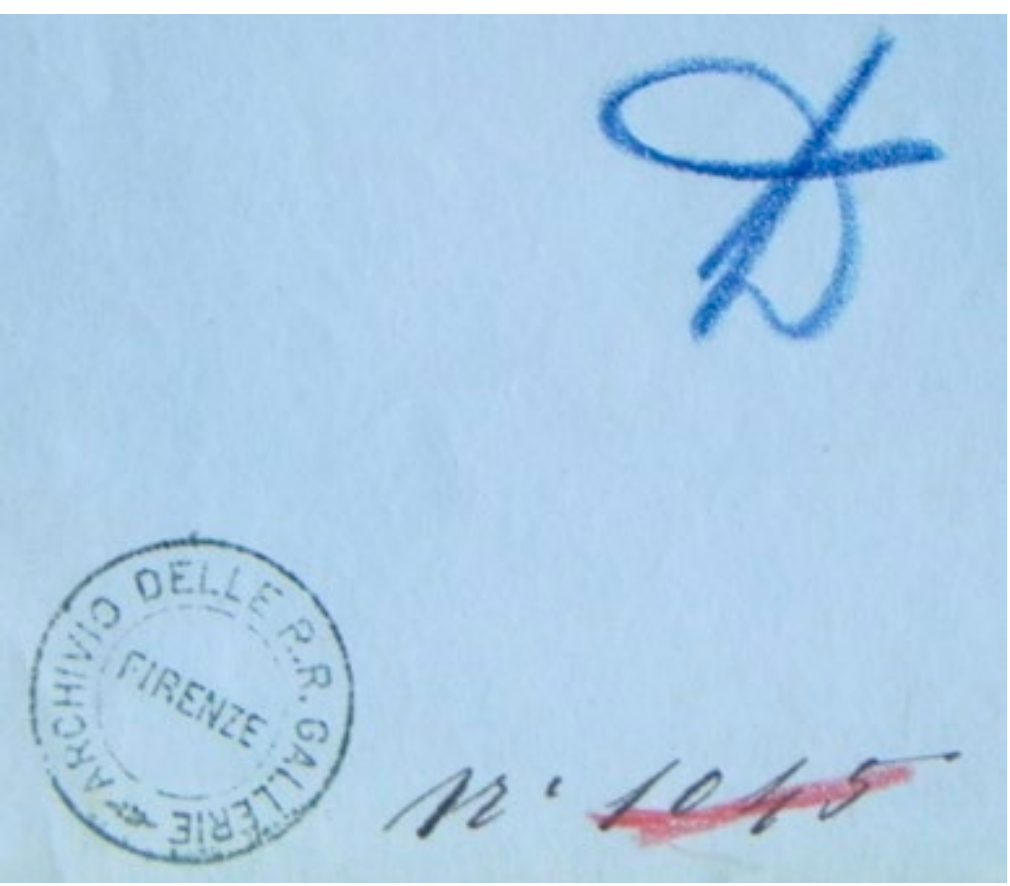




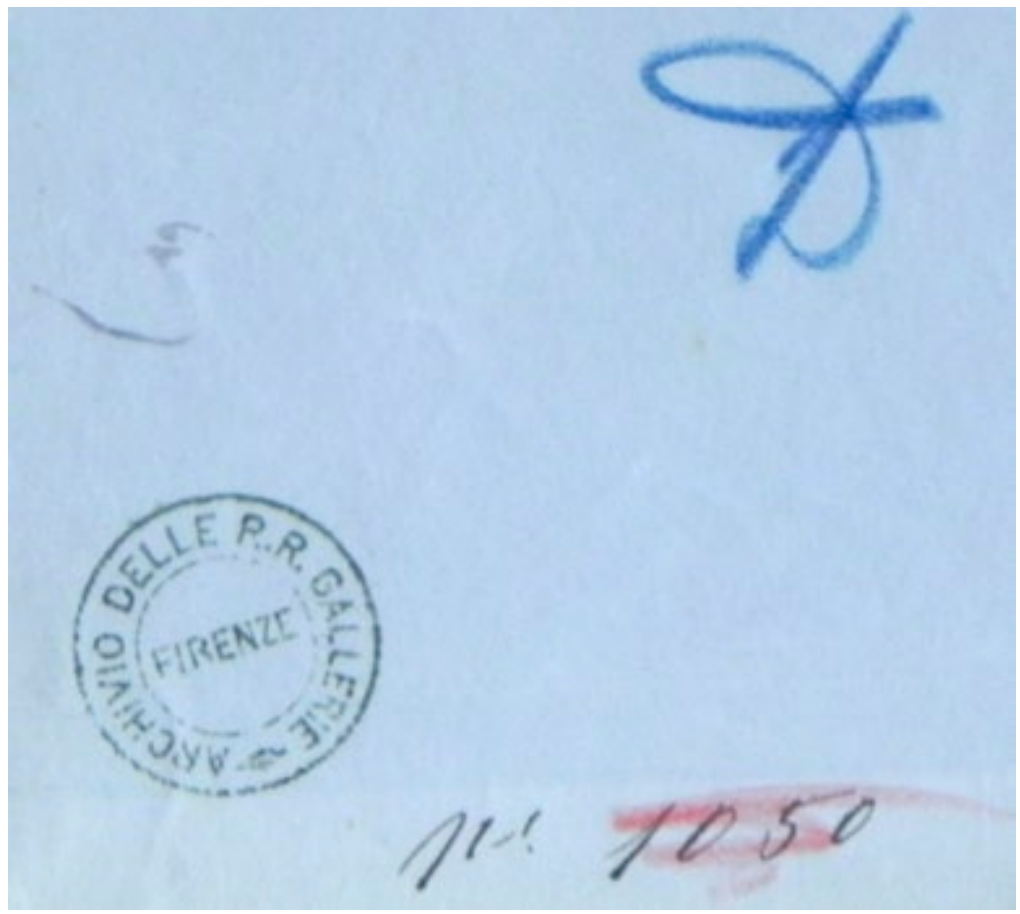

\section{Brogi,}

Raffaello: dettaglio della Madonna del Baldacchino, 1878.

Stampa all'albumina, $25,1 \times 19,4 \mathrm{~cm}$, dettaglio del verso.

Firenze, ASGF, dossier "Raffaello Inv. Palatina".

Riproduzione su concessione del Ministero per i Beni e le Attività

Culturali

\section{6}

\section{Brogi,}

Raffaello: particolare della Madonna del Baldacchino, 1878.

Stampa all'albumina, $25,1 \times 19,4 \mathrm{~cm}$, dettaglio del verso.

Firenze, ASGF, dossier "Raffaello Inv. Palatina". Riproduzione su concessione del Ministero per i Beni e le Attività Culturali

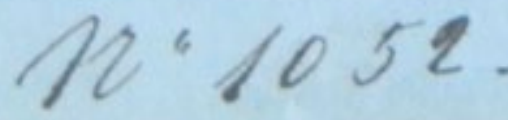


che, con il numero d'ordine "7905", entra in catalogo successivamente al 1878 ed è ancora presente nel $1903^{-36}$.

La fotografia che riporta il numero "1047" è la riproduzione del dipinto Madonna della Seggiola; con il numero in didascalia editoriale "2905a" entra anch'essa in catalogo successivamente al 1878 e si attesta nel catalogo di inizio Novecento ${ }^{-37}$. Il numero "1048" compare sul verso della fotografia della Madonna del Granduca (fig. 2). Entrambe le fotografie sono segnate sul verso dal timbro dell'“Archivio delle RR. Gallerie di Firenze" e da quello dell'“Ufficio Ricerche”.

Le fotografie con numero manoscritto "1050" e "1052" (figg. 5-6) corrispondono a due dettagli del dipinto Madonna del Baldacchino: la prima è il particolare della Madonna in trono col bambino, che di nuovo porta sul verso il timbro dell'“Archivio delle Regie Gallerie” e lo stesso vale per la seconda, il particolare dei due angioletti che leggono il cartiglio con la musica ai piedi del baldacchino. Le due fotografie portano, rispettivamente, il numero d'ordine "2937" e "2936a": entrambe entrano in catalogo nel $1878^{-38}$. L'ultima fotografia (fig. 7) reca sul verso una didascalia manoscritta a inchiostro che comprende il numero d'inventario: "Brogi No 1053 Ritratto di donna Uffizi Raffaello". Vi figurano altri quattro timbri a inchiostro, relativi alla "Regia Galleria di Firenze", all'“Archivio delle RR. Gallerie di Firenze”, alla "Regia Soprintendenza all'arte medioevale e moderna per la Toscana (Ufficio Catalogo) Firenze" e all'“Ufficio Ricerche”. Tra varie note manoscritte, in diversa grafia e corrispondenti a momenti differenti, una didascalia a matita, evidentemente più recente, dichiara "Raffaello, La muta, Urbino" -39.

Come accennato, dato che i numeri manoscritti presenti qui considerati non appartengono né al numero d'inventario del dipinto nel museo, né al numero d'ordine del catalogo di vendita, ho supposto che essi possano rinviare all'inventario delle fotografie entrate al museo e che siano stati assegnati al momento dell'ingresso della fotografia o, con maggiore probabilità, al momento dell'organizzazione dell'Archivio Fotografico da parte di Corrado Ricci e della conseguente inventariazione delle fotografie precedentemente acquisite.

Per legittimare questa ipotesi è necessario soffermarsi sulla nascita dell'archivio. Nel 1904, la stampa estera annunciava l'istituzione di un archivio fotografico nelle Gallerie di Firenze, innovativa operazione già sperimentata a Milano col Ricetto Fotografico della Pinacoteca di Brera ${ }^{40}$. Scriveva il "Boston Evening Transcript" il 16 novembre 1904, a proposito delle iniziative attuate da Corrado Ricci agli Uffizi:

\footnotetext{
As for the reference library, not only shall it contain all kinds of books, periodicals and pamphlets relating to art, but will supplemented by an archive of photographic documents, reproductions of works of art the world over [...] Several thousands of photographic documents are already being classified, and probably before next spring a part at least of the library and archives will be in working order $-{ }^{41}$.
} 


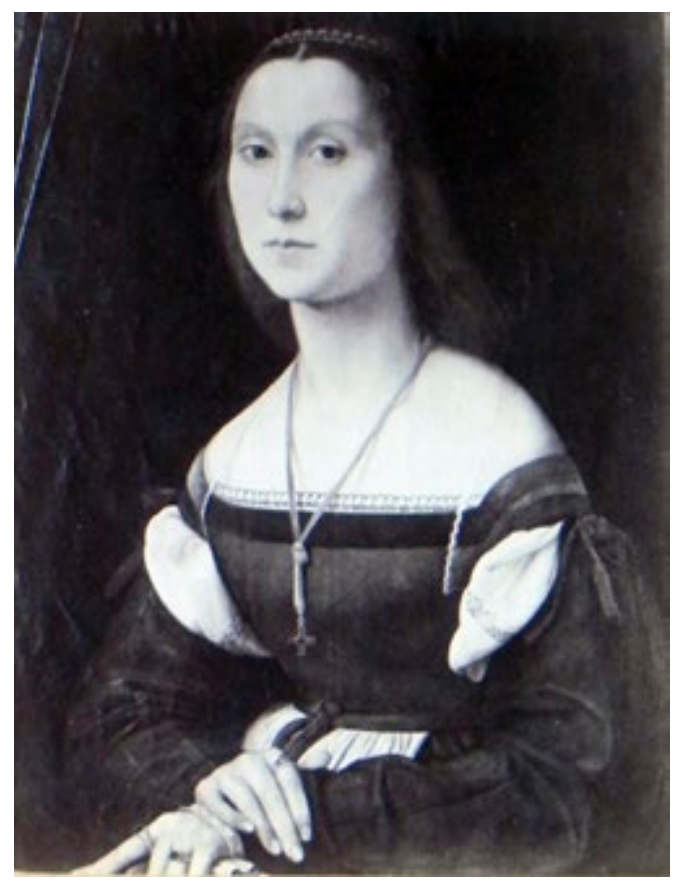

a.

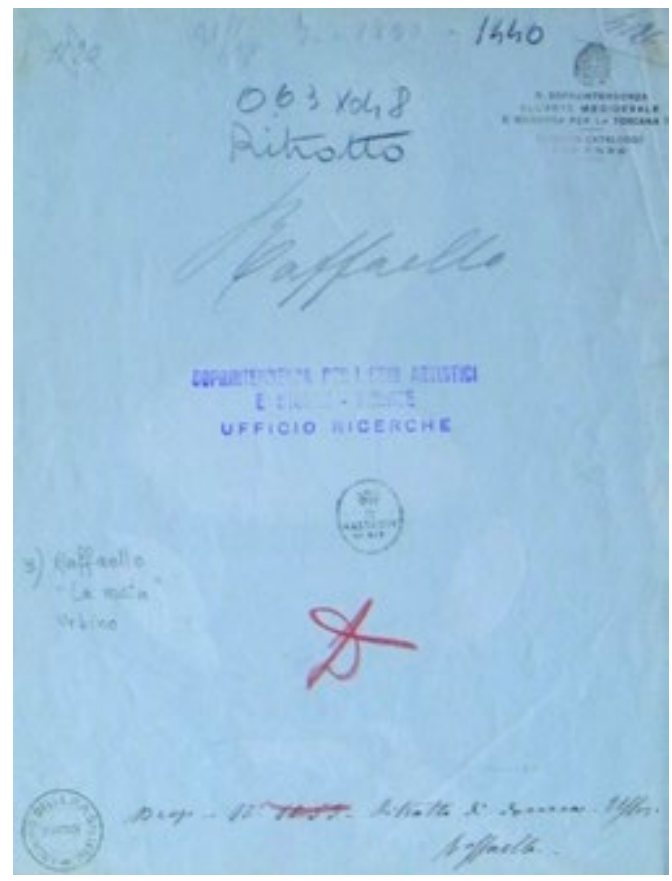

b.
07

\section{Brogi,}

a. Raffaello: La muta, 1878.

Stampa all'albumina,

$24,4 \times 18,5 \mathrm{~cm}$, recto.

Firenze, ASGF, dossier

"Raffaello Inv. Palatina".

Riproduzione su

concessione del Ministero

per i Beni e le Attività

Culturali

b. Verso della fotografia

con timbri e note

manoscritte 
Due timbri a inchiostro rinvenuti su fotografie dell'Archivio Storico delle Gallerie Fiorentine (1904). Riproduzione su concessione del Ministero per i Beni e le Attività Culturali

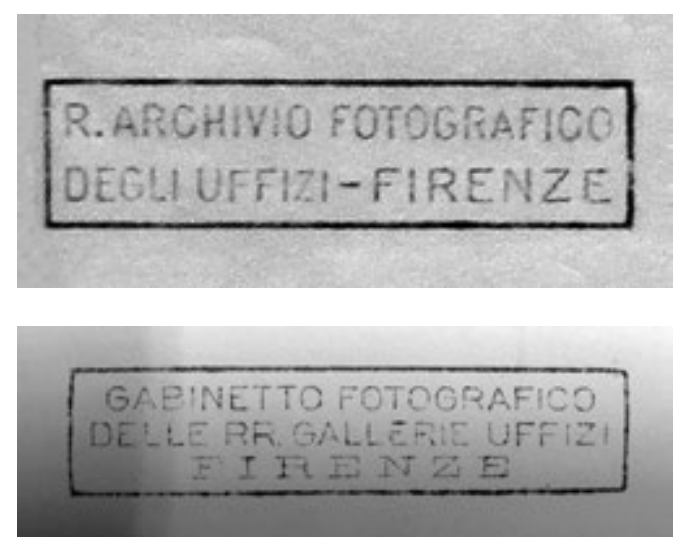

La notizia della nuova direzione delle Gallerie Fiorentine era giunta fin negli Stati Uniti e ancor prima in Europa, dove già in gennaio si commentava:

Monsieur Ricci s'efforcera surtout d'obtenir de la maison du Roi quelques nouvelles galeries. Dans l'une d'elles il placera des portraits actuellement relégués dans les combles, dans trois autres les dessins qui seront montrés au public par des expositions successives; et, dans la dernière, des collections de photographie $-{ }^{42}$.

Si può ben pensare che le fotografie depositate dalla ditta Brogi facessero parte di queste collezioni fotografiche, considerato che nel secondo Ottocento la ditta aveva depositato direttamente alle Regie Gallerie circa seicento stampe ${ }^{-43}$.

Un'ultima traccia presente su alcune delle fotografie dello schedario dell'Archivio Storico delle Gallerie Fiorentine confermerebbe l'esistenza, nell'ambito di una stessa entità, di due diverse sezioni organizzate e articolate secondo precise e distinte funzioni: l'Archivio fotografico e il Gabinetto fotografico. A essi corrispondono i rispettivi timbri (fig. 8), che possono trovarsi anche insieme sulla stessa fotografia, presumendo che sia stata prodotta dal Gabinetto e conservata in Archivio storico -44.

A questo proposito, era stato lo stesso Ricci a dichiarare che il suo progetto prendeva avvio come una raccolta: nel documento a stampa del novembre del 1903 (pubblicato con grande solerzia considerando che l'incarico di Direttore gli era stato assegnato in ottobre), Ricci proponeva la costituzione di un archivio fotografico, ovvero di un luogo di raccolta di materiali fotografici a uso di artisti e studiosi:

In tale deposito si raccoglierebbero, nel maggior numero possibile, fotografie d'opere d'arte, di luoghi, d'avvenimenti, di persone ragguardevoli in ogni campo dello scibile [...]. Ora domando: perché non istituire agli Uffizi un Archivio fotografico, dove un materiale, vantaggioso tanto agli artisti quanto ai dotti, trovi una sede pubblica e un ordinamento 
pratico, per autori e per materie? Né si creda che l'istituzione presenti grandi difficoltà. Essa intanto può trovar sede nel Palazzo degli Uffizi vicino alle Gallerie, alla Biblioteca, e all'Ufficio Regionale per la conservazione dei Monumenti della Toscana: ed avere il suo ispettore e il suo orario. Ma per formare una prima raccolta di fotografie con le principali collezioni e perché questa possa ampliarsi, ricorro all'aiuto della S. V. Ill.ma, aiuto di consiglio, d'oblazioni, di doni di fotografie, ritraenti qualsiasi oggetto artistico o luogo o monumento ${ }^{-45}$.

Si può ben pensare che il Gabinetto di produzione di fotografie sia stato istituito contestualmente o a seguito della fondazione dell'Archivio, come conseguenza fisiologica della sentita necessità di dotare i musei di un patrimonio visivo a servizio dello studio non solo delle opere presenti nelle collezioni, ma anche di quelle presenti nel territorio e delle cose d'arte nel senso più ampio del termine ${ }^{-46}$.

Fra Seicento e Settecento, il collezionismo del gran principe Ferdinando de' Medici è stato definito policentrismo artistico e teso a modernizzare l'ambiente fiorentino con acquisti che talvolta non si rivelarono capolavori, data la smania di attribuzione ai grandi artisti. Il caso più significativo è quello dei ben 19 dipinti attribuiti a Tiziano, allora copiati e incisi, dei quali, in verità, oggi soltanto due sono confermati dalla critica: l'Adorazione dei pastori e la Venere di Urbino ${ }^{-47}$. Lo slancio che aveva accompagnato la committenza di campagne incisorie nei musei era stato segnato senza dubbio dal desiderio dei granduchi collezionisti di ostentare la ricchezza dei propri patrimoni artistici, in funzione di un riconoscimento del loro valore non soltanto culturale ma anche politico con lo scopo di "riprodurre in incisione per far conoscere dipinti e disegni” -48 .

Alla metà dell'Ottocento, invece, quando la fotografia entrò ufficialmente nelle Gallerie, non venne strumentalizzata dai direttori dei musei così come lo era stata l'incisione da parte del gran principe Ferdinando e di Cosimo III nel secolo precedente. Al contrario, la fotografia incontrò una certa resistenza, fino al veto imposto dal Ministero della Pubblica Istruzione di riprodurre i dipinti delle collezioni pubbliche fiorentine ${ }^{-49}$. Tale divieto - su cui lo stesso Ministero, come abbiamo visto, ebbe un ripensamento promulgando il 14 ottobre del 1867 il decreto che regolamentava le attività fotografiche nei musei pubblici - era dettato dalla volontà del Ministro di non creare un precedente problematico. Da una parte, il divieto mirava infatti a salvaguardare l'incolumità dei quadri, che sarebbe stata messa a rischio se si fosse iniziato a staccarli sovente dalle pareti per metterli in condizione di luce migliore per essere fotografati. Dall'altra parte, l'intento era quello di preservare la pratica della copia pittorica delle opere: lasciando libero accesso ai fotografi, si sarebbe avuto infatti un "danno irreparabile di molti artisti che vivono in sul copiare" ${ }^{50}$. Come possiamo capire, il rapporto tra i fotografi e gli enti preposti alla tutela del patrimonio era complesso, al punto che tranne puntuali occasioni, tra gli anni Sessanta e Settanta, 
la fotografia entrò nei musei fiorentini non per volere dei conservatori, che probabilmente la guardavano con sospetto, ma grazie all'iniziativa privata degli stabilimenti fotografici, che condussero le proprie campagne in piena autonomia.

Nei fatti, era mutato il concetto di museo, ormai divenuto pubblico, e si era trasformata la funzione che i patrimoni artistici erano chiamati a svolgere nella società. Il ruolo delle pinacoteche non consisteva più nello sfoggio di ricchezza e potere, così emblematico nei patrimoni d'arte, ma nell'essere specchio dell'identità nazionale, segno della Storia e del primato artistico italiano. Dopo l'Unità del paese, la funzione conservativa, di tutela e didattica dei musei prendeva così il sopravvento rispetto all'esibizione dei poteri del singolo sovrano.

In altre parole, rispetto al Settecento, era venuta meno la committenza. A mio parere, questo vuoto restituisce una delle motivazioni per cui inizialmente ai fotografi fu rifiutato o quantomeno ostacolato l'ingresso nelle raccolte museali. Questi ostacoli non scaturivano solo da questioni tecniche e di valutazione della qualità dell'immagine, o da una questione riferita alla sterile meccanicità della camera oscura rispetto all'esecuzione della copia eseguita dall'artista, ma anche dal fatto che sul momento non si avvertiva la necessità di acquisire uno strumento di riproduzione delle opere del museo, seppur capace di accompagnare queste istituzioni nella modernità. Semmai, vi era la necessità di proteggere un patrimonio riconosciuto come bene nazionale e tutelare il mercato tradizionale delle copie.

Ciò nonostante, la volontà dei fotografi di far circolare sul mercato riproduzioni di determinati soggetti artistici a uso e consumo di artisti, conoscitori e semplici viaggiatori prevaricò sui timori e sulle reticenze degli organi preposti alla tutela delle collezioni pubbliche. Gli stabilimenti fotografici, con il loro sistema di produzione nell'ambito della fotografia d'arte, trovarono a Firenze, in una figura lucida e recettiva come Corrado Ricci, colui che alla volta del nuovo secolo seppe cogliere ed enfatizzare la funzione pubblica della fotografia a servizio degli studi, dell'arte e del moderno ruolo del museo ${ }^{-51}$.

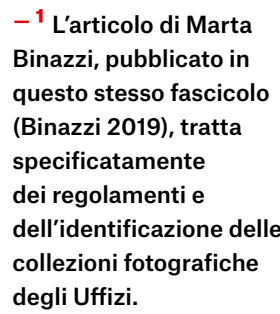

- ${ }^{1}$ L'articolo di Marta Binazzi, pubblicato in questo stesso fascicolo (Binazzi 2019), tratta specificatamente dei regolamenti collezioni fotografiche degli Uffizi.

$-{ }^{2}$ La notizia della
circolare ministeriale è
presente in una lettera
manoscritta da parte del
Ministero della Pubblica
Istruzione al Direttore delle
Regie Gallerie rilevata
nell'Archivio Storico
delle Gallerie Fiorentine.

Cfr. \#MPI 1867a. Come riportato da Chiara Migliorini (Migliorini 1994, p. 46), la circolare era pervenuta anche all'Accademia di Belle Arti di Firenze.

$-{ }^{3}$ Sul tema dei cataloghi commerciali, oltre a 
Tomassini 2003, si veda il recente volume Cavanna / Mambelli 2019 e al suo interno, sul tema specifico della fotografia agli Uffizi, cfr. Naldi 2019. $-{ }^{4}$ Sulla storia degli archivi fotografici delle Soprintendenze si rimanda almeno agli atti di un importante convegno: Spiazzi / Majoli / Giudici 2010, oltre che a: Stanzani / Orsi / Giudici 2001 per il caso bolognese. Per le vicende del Gabinetto fotografico di Giovanni Gargiolli cfr. i testi in Marsicola 2014. - 5 Migliorini 1994, Maffioli 2017.

$-{ }^{6}$ Ho effettuato lo spoglio durante le ricerche per il dottorato di ricerca (2014-2017), mirate a individuare i documenti delle attività dei fotografi e dei copisti, inizialmente connesse, individuando circa $\mathbf{3 0 0}$ fascicoli, di cui la metà concerne le attività fotografiche.

$-{ }^{7}$ Nella storiografia si annoverano casi noti di non ottemperanza dell'obbligo da parte dei fotografi, come quello della Maison Braun, che sin dal 1866 organizzò sistematicamente campagne fotografiche nei maggiori musei europei e che entrò in contrasto con il Louvre nel 1877, dal momento che lo Stato francese aveva predisposto il primo regolamento sulle attività fotografiche nei musei solo nel 1872: “Peu à peu, un conflit naît entre Braun et l'administration des musées, car l'éditeur néglige très régulièrement de déposer aux archives du musée les deux épreuves des chaque clichés exécuté, tel que l'exige le règlement" (Renié 1999, p. 105).
$-{ }^{8}$ Le fotografie oggetto di questo studio non sono state censite nei diversi interventi di Marilena Tamassia (2014a; 2014 c; 2012; 2011; 1996).

$-{ }^{9}$ Per la maggior parte queste fotografie risultano sciolte e presentano una ricca testimonianza del proprio percorso sul verso: numerosi sono i timbri e le note manoscritte, cui accenneremo in seguito, che meriterebbero uno studio dedicato per ricostruire la cronologia dei luoghi amministrativi che le hanno accolte nel corso del tempo. Ringrazio Francesca Moschi per il supporto alle ricerche e per avermi indicato la presenza di materiali fotografici all'interno dello schedario.

- 10 Nel 1958, il patrimonio di $\mathbf{5 0 . 0 0 0 ~ l a s t r e ~ e ̀ ~ c o n f l u i t o ~}$ nell'Archivio Fratelli Alinari. Per la bibliografia su Giacomo Brogi si rimanda a Maffioli / Bietoletti 2014, p. 333; Maffioli 1999; Berselli 1994; Silvestri 1994; Tempesti 1994; Arbib 1882. II regesto dei cataloghi si trova in buona parte in Maffioli 1996.

- ${ }^{11}$ La notizia è riportata nella biografia del fotografo in calce a un catalogo di mostra (cfr. Maffioli / Bietoletti 2014 p. 333).

-12 Brogi 1865. Un esemplare è conservato presso la Biblioteca Nazionale Centrale di Firenze.

$-{ }^{13}$ Per il concetto di "vocazione enciclopedica", cfr. Heilbrun 2003, p. 68.

- ${ }^{14}$ Sul tema francese cfr. Boyer 2004, Tome II; Boyer 1998, pp. 82-91 e Font-Réaulx (de) 2012. Per I'attività iniziale di Brogi: Maffioli 1996, p. 244.
- ${ }^{15}$ Brogi 1874 (ho consultato la copia conservata presso la Biblioteca degli Intronati di Siena).

- 16 \#Brogi 1874.

- ${ }^{17}$ Cfr. \#DRRG 1875.

- ${ }^{18}$ Brogi 1876a, Brogi

1876b e Brogi 1876c. I

cataloghi di Brogi sono

tutti editi in lingua

francese, tranne quello del 1865.

- 19 In calce alla lettera specifica le quantità: 14 copie in formato extra $(20 \times 25 \mathrm{~cm})$ e 2 copie in formato sopra extra $(28 \times 38$ cm) (cfr. \#Brogi 1877). - 20 \#MPI 1878.

$-{ }^{21}$ Brogi 1878. I soggetti tratti dai dipinti della Galleria Palatina e inseriti per la prima volta in catalogo sono in tutto 244 . - 22 \#DRRG 1879b. $-{ }^{23}$ Cfr. \#DRRG 1879c.

Nello stesso fascicolo ci sono altre quattro lettere circolate negli stessi giorni tra Brogi, la Direzione delle Gallerie e il Ministero riguardo la richiesta di permesso di fotografare I'affresco di Domenico Ghirlandaio nel refettorio del Convento di Ognissanti cui il Ministero dà l'avallo per la licenza (cfr. \#Brogi 1879; \#DRRG 1879a; \#MPI 1879; \#DRRG 1879d).

- 24 Madonna della

Seggiola, Madonna del

Granduca, Madonna del

Baldacchino, Concerto di Giorgione da confrontare con Brogi 1878, p. 27,

18,30 . Questi soggetti saranno ancora in catalogo all'inizio del Novecento con lo stesso numero d'ordine (cfr. Brogi 1903, pp. 38, 48, 53).

-25 In questa sede non ci addentriamo nello specifico dei timbri rilevati sugli oggetti fotografici poiché è ancora in corso 
un'indagine dedicata che potrà chiarire meglio le pertinenze dei vari uffici competenti che, nel tempo, si sono succeduti, e gli spostamenti che le fotografie hanno subito nei vari archivi all'interno delle gallerie.

- 26 \#Filza 1880.

-27 La pratica relativa è costituita da cinque lettere (cfr. \#Sovrintendente Bossi Segrez 1881). Nello stesso fascicolo è presente un'altra richiesta: per le statue di Michelangelo dei monumenti ai Medici, per gli affreschi della cappella degli Spagnoli in Santa Maria Novella e per un disegno del quale accludono una fotografia, commissionatogli dal bibliotecario dell'Imperatore di Germania (cfr. \#Brogi 1881).

- ${ }^{28}$ Ci limitiamo a questo punto a citare i fascicoli in cui si attesta l'attività di Brogi senza entrare nel merito delle singole pratiche, cosi da fornire un'idea delle consistenze: \#Riproduzioni fotografiche 1882; \#Brogi 1883; \#Filza 1885; \#Brogi 1885; \#Filza 1886; \#Riproduzioni 1887; \#Riproduzioni 1888; \#Filza 1889.

-29 Sono certamente numerosi i contributi teorici che applicano l'approccio Material turn allo studio della fotografia, di cui Elizabeth Edwards è il principale referente $\mathrm{e}$ della quale, per brevità, citeremo: Edwards 2015 e Edwards 2004. Per i contributi collettivi ricordiamo Caraffa 2011. In questo frangente scegliamo di menzionare più nello specifico uno dei testi di Costanza Caraffa che consideriamo tra i più prossimi alla metodologia applicata. Scrive, infatti, la studiosa: "documentary photographs are documents not only in relation to the object they are intended to document, but also - precisely because photography is not neutral - in relation to a whole series of other aspects that are, whether intentionally or not, registered in them: they are documents, for example, of the contemporary level of the technological development of photography, of the interest of research in a particular theme in a particular period, of different practices of displaying works of art in photographs, of the attributive history of a particular work of art as annotated on the mount of its photograph" (Caraffa 2013, p. 824).

- 30 Timbro a inchiostro

"Fototeca Gamba". Carlo Gamba (Firenze 1879 - Ivi 1963), storico dell'arte e collezionista, partecipò al progetto di riordino di Corrado Ricci delle Regie Gallerie Fiorentine (1901-1906), di cui divenne ispettore onorario nel 1907 (cfr. Ginori Lisci 1979;

Bigazzi / Todros 2001; Masini 2015).

- 31 Filippo Rossi

(Firenze, 1892-1974) fu soprintendente a Firenze dal 1927 al 1962 (cfr. Masini 2015).

-32 “What do these

photographs actually document?" (cfr. Caraffa 2013, p. 825).

- 33 Riportiamo queste diciture a titolo esemplificativo, ribadendo che il necessario lavoro di identificazione dei timbri per ricondurli a una determinata scansione cronologica è ancora da compiere.

-34 Nel catalogo del 1878, tra le fotografie tratte dagli originali della Galleria Palatina compaiono 18 opere e 21 soggetti, proposti nei formati extra, super-extra ed extragrande (cfr. Brogi 1878, pp. 27-28 e 46).

- 35 Purtroppo la copia

del catalogo Brogi edito nel 1889 , che segue quello del 1878 conservato presso la Biblioteca Marucelliana, non era disponibile per la consultazione al momento della stesura del testo. $\mathrm{Mi}$ atterrò pertanto al catalogo ancora successivo a nostra disposizione, quello del 1903 (cfr. Brogi 1903, p. 48).

-36 Ibid.

-37 lbid.

- 38 Cfr. Brogi 1878, p. 27.

- 39 Si ricorda che questo ritratto di Raffaello, dopo essere stato trasferito da Palazzo Pitti e da villa di Poggio a Caiano, era entrato agli Uffizi nel 1773. Nel 1927 era stato donato alla Galleria Nazionale delle Marche, dove si trova tutt'oggi, come opera rappresentativa del maestro urbinate.

-40 Sul tema si rimanda alla bibliografia specifica: Serena 2017; Ghibaudi 2012; Bonetti 2010 e Miraglia / Ceriana 2000. - 41 Cfr. \#Bernardy 1904. - 42 Les musées 1904. - 43 Per la consistenza dei lasciti della ditta Brogi si rimanda all'articolo di Marta Binazzi edito in questo stesso numero di "RSF. Rivista di studi di fotografia".

-44 Timbri sul verso di una fotografia conservata nel faldone “Gallerie. Piante e Vedute. Recenti 
Acquisti", Materiale

Iconografico, Serie IX, sottoserie Firenze / Musei

n. 170, Archivio Giovanni

Poggi - Firenze, ASGF.

- 45 \#Ricci 1903. II

documento di Ricci è stato interamente trascritto $\mathrm{e}$ pubblicato in Tamassia 2011, nota 1, p. 73 e ancora in Tamassia 2014c, nota 3 , pp. 87-88.

- 46 || Gabinetto

Fotografico è stato oggetto di studi da parte di Marilena Tamassia per cui si rimanda ai suoi contributi: Tamassia 2014a; Tamassia 2014b; Tamassia 2014c; Tamassia 2012; Tamassia 2011; Tamassia 1996.
- ${ }^{47}$ Cfr. Borroni Salvadori 1982.

- ${ }^{48}$ Come recita il titolo

di lbid.

- 49 II MPI aveva imposto

al DRRG di vietare

a Giacomo Brogi di

fotografare nella Galleria

degli Uffizi nel luglio del

1867. Tuttavia, la direzione

del museo concesse sia

a Brogi che agli Alinari il

permesso di riprodurre

i disegni degli antichi

maestri ma in numero

limitato, continuando a

vietare la riproduzione

dei dipinti a chiunque (cfr.

\#MPI 1867b).

- 50 lbid.

- ${ }^{51}$ Ringrazio l'Archivio

Storico delle Gallerie
Fiorentine per la

disponibilità nel mettere

a disposizione il materiale

oltre che per la preziosa

assistenza durante le

ricerche e il Ministero

per i Beni e le Attività

Culturali per aver concesso

la pubblicazione delle

fotografie. L'articolo,

redatto nell'estate nel

2019, ha trovato conferma,

in alcune sue ipotesi, nel

ritrovamento da parte

della Soprintendenza del

Regio Archivio Fotografico

degli Uffizi. L'Archivio è

attualmente oggetto di un

progetto d'inventariazione supervisionato dalla

dott.ssa Valentina

Conticelli.

Arbib 1882 Giacomo Arbib, Di Giacomo Brogi fotografo, la sua vita e le sue opere, Roma, Roma Artistica, 1882.

Berselli 1994 Silvia Berselli, Le 'specialità artistiche' della casa Giacomo Brogi. I grandi formati per la riproduzione delle opere d'arte, in "AFT. Rivista di storia e fotografia", n. 20, 1994, pp. 4-5.

Bigazzi / Todros 2001 Isabella Bigazzi / Rossella Todros (a cura di), Lo sport in passerella. Figurini sportivi nella collezione Gamba, catalogo della mostra (Firenze, Biblioteca Marucelliana, 2001), Firenze, Nardini, 2001.

Binazzi 2019 Marta Binazzi, Fotografie e istituzioni museali: il sistema della doppia copia e l'accumulo dei fondi. Le Regie Gallerie di Firenze, 1860-1906, in “RSF. Rivista di studi di fotografia", 10, 2019, pp. 10-35.

Bonetti 2010 Maria Francesca Bonetti, I/ collezionismo fotografico nelle istituzioni: una necessità o una scelta?, in Francesco Faeta / Giacomo Daniele Fragapane (a cura di), Forme e modelli. La fotografia come modo di conoscenza, atti del convegno (Noto, 2010), Roma-Messina, Corisco Edizioni, 2010, pp. 53-62, disponibile anche in <http://www.coriscoedizioni.it/wp-content/ uploads/2013/10/Forme-e-Modelli.-La-fotografia-come-modo-di-conoscenza. pdf > (15.07.2019).

Borroni Salvadori 1982 Fabia Borroni Salvadori, Riprodurre in incisione per far conoscere dipinti e disegni: il Settecento a Firenze, in "Nouvelles de la République des lettres", n. 1, 1982, pp. 7-71.

Boyer 1998 Laure Boyer, Adolphe Braun 1812-1877 et la reproduction photographique des œuvres d'art, thèse Université de Strasbourg, sous la direction de Eric Michaud, 1998 (consultata presso il Cabinet des estampes et de la photographie, BNF, Paris). 
Boyer 2004 Laure Boyer, La photographie de reproduction d'oeuvres d'art au XIX siècle en France 1839-1919, thèse de doctorat Université de Strasbourg, sous la direction de Roland Recht, 2 voll., 2004 (consultata presso la Bibliothèque du Musée d'Orsay, Paris).

Brogi 1865 Giacomo Brogi, Catalogo della Pinacoteca Universale. Grandiosa raccolta fotografica d'insigni dipinture, disegni ecc. edita in tre differenti formati, Firenze, Tip. G. Barbera, 1865.

Brogi 1874 Catalogo delle fotografie riprodotte dagli originali. Quadri, Affreschi, Disegni, ecc., Firenze, Stabilimento Giuseppe Civelli, 1874.

Brogi 1876a Giacomo Brogi, Reproductions d'après les originaux de la Pinacothèque de Milan (Palais Brera), II livraison, Florence, Établissement Joseph Civelli, 1876.

Brogi 1876b Giacomo Brogi, Reproductions d'après les originaux de la Galerie de Florence (Uffizi), III livraison, Florence, Établissement Joseph Civelli, 1876.

Brogi 1876c Giacomo Brogi, Reproductions d'après les originaux du Musée de St. Marc de Florence, IV livraison, Florence, Établissement Joseph Civelli, 1876.

Brogi 1878 Giacomo Brogi, Catalogue des photographies publiées par la maison Giacomo Brogi de Florence, première partie tableaux, fresques, dessins, Florence, Établissement Civelli, 1878.

Brogi 1903 Giacomo Brogi, Catalogue des reproductions en photographies publiées par la Maison Giacomo Brogi, Florence, 1903.

Caraffa 2011 Costanza Caraffa (a cura di), Photo Archives and the Photographic Memory of Art History, atti delle conferenze (Londra e Firenze, 2009), BerlinMünchen, Deutscher Kunstverlag, 2011.

Caraffa 2013 Costanza Caraffa, Documentary Photographs as Objects and Originals, in Ulrich Großmann / Petra Krutisch (a cura di), The Challenge of the Object, atti del convegno (Norimberga, 2012), t. 3, Nürnberg, Verlag des Germanischen Nationalmuseums, 2012, pp. 824-827.

Cavanna / Mambelli 2019 Pierangelo Cavanna / Francesca Mambelli, Un patrimonio da ordinare: $i$ cataloghi a stampa dei fotografi, atti del convegno (Bologna 2018), Bologna, Fondazione Federico Zeri, 2019.

Edwards / Hart 2004 Elizabeth Edwards / Janice Hart (a cura di), Photographs Objects Histories. On the Materiality of Images, London, Routledge, 2004.

Edwards / Morton 2015 Elizabeth Edwards / Christopher Morton (a cura di), Photographs, Museums, Collections. Between Art and Information, London, Bloomsbury, 2015.

Font-Réaulx (de) 2012 Dominique de Font-Réaulx, Peinture \& Photographie, les enjeux d'une rencontre, 1839-1914, Paris, Flammarion, 2012.

Ghibaudi 2012 Cecilia Ghibaudi, Il ricetto fotografico della Pinacoteca di Brera: nascita e formazione di un Archivio Fotografico nell'Ottocento, in Maria Fratelli / Francesca Valli (a cura di), Musei nell'Ottocento alle origini delle collezioni pubbliche lombarde, atti delle giornate di studio (Milano, 2010), Torino, Allemandi, 2012, pp. 425-436.

Ginori Lisci 1979 Leonardo Ginori Lisci, Count Carlo Gamba, a Florentine Gentleman of Former Times, in "Apollo", n. 110, 1979, pp. 108-113.

Heilbrun 2003 Françoise Heilbrun, Alinari e Nègre, in Arturo Carlo Quintavalle / Monica Maffioli (a cura di), Fratelli Alinari fotografi a Firenze. 150 anni che illustrarono il mondo 1852/2002, Firenze, Alinari, 2003, pp. 57-69. 
Les musées 1904 Les musées de Florence, in "Gazette de Liège" 18 janvier 1904, ritaglio consultato in Serie Studi Busta 19, Fascicolo 123, Sotto fascicolo 3, Fondo Corrado Ricci, Biblioteca Classense, Ravenna.

Maffioli 1996 Monica Maffioli, /l BelVedere. Fotografi e architetti nell'Italia dell'Ottocento, Torino, SEI, 1996.

Maffioli 1999 Monica Maffioli, La stereoscopia nella produzione degli stabilimenti fotografici dei Fratelli Alinari e di Giacomo Brogi, in "AFT. Rivista di storia e fotografia", n. 30, 1999, pp. 36-40.

Maffioli 2017 Monica Maffioli, "Del metodo del fare e del metodo del vedere": la fotografia all'Accademia di Belle Arti di Firenze nella seconda metà dell'Ottocento, in Cristina Frulli / Francesca Petrucci (a cura di), L'Accademia di Belle Arti di Firenze negli anni di Firenze capitale 1865-1870, atti del convegno (Firenze, 2015), Firenze, Edizioni dell'Assemblea, Consiglio Regionale Toscana, 2017, pp. 337-361.

Maffioli / Bietoletti 2014 Monica Maffioli / Silvestra Bietoletti (a cura di), Ri-conoscere Michelangelo. La scultura del Buonarroti nella fotografia e nella pittura dall'Ottocento a oggi, catalogo della mostra (Firenze, Galleria dell'Accademia, 2014), Firenze, Giunti, 2014.

Marsicola 2014 Clemente Marsicola (a cura di), Il viaggio in Italia di Giovanni Gargiolli. Le origini del Gabinetto Fotografico Nazionale 1895-1913, catalogo della mostra (Roma, Istituto Centrale per il Catalogo e la Documentazione, 2014-2015), Roma, ICCD, 2014.

Masini 2015 Marta Masini, Archivi in biblioteca. Le carte di Filippo Rossi e del Conte Carlo Gamba, Tricase, Youcanprint Self-Publishing, 2015.

Migliorini 1994 Chiara Migliorini, La fotografia come modello. L'Accademia di Belle Arti di Firenze, in "AFT. Rivista di storia e fotografia", n. 19, 1994, pp. 43-51.

Miraglia / Ceriana 2000 Marina Miraglia / Matteo Ceriana (a cura di), Brera 1899, un progetto di fototeca pubblica per Milano. II "ricetto fotografico" di Brera, catalogo della mostra (Milano, Pinacoteca di Brera, 2000), Milano, Electa, 2000.

Naldi 2019 Chiara Naldi, I dipinti "fuori luogo" nel catalogo Alinari del 1863. Criteri di selezione nella prima campagna fotografica agli Uffizi, in Pierangelo Cavanna / Francesca Mambelli, Un patrimonio da ordinare: i cataloghi a stampa dei fotografi, atti del convegno (Bologna 2018), Bologna, Fondazione Federico Zeri, 2019, pp. 287-311.

Renié 1999 Pierre-Lin Renié, Braun vs Goupil et quelques autres histoires. La photographie au Musée du Louvre au XIX siècle, in "État des lieux", n. 2, 1999, pp. 97-151.

Serena 2017 Tiziana Serena, Le musée d'art comme lieu d'autorité pour l'archive photographique. Le cas italien au tournant du XX siècle, in Musées de photographies documentaires, num. mon. di “Transbordeur", n. 1, 2017, pp. 50-61.

Silvestri 1994 Silvia Silvestri, Lo Studio Brogi a Firenze. Da Giacomo Brogi a Giorgio Laurati, in "AFT. Rivista di storia e fotografia", n. 20, 1994, pp. 9-32.

Spiazzi / Majoli / Giudici 2010 Anna Maria Spiazzi / Luca Majoli / Corinna Giudici (a cura di), Gli archivi fotografici delle soprintendenze. Tutela e storia. Territori veneti e limitrofi, atti del convegno (Venezia, 2008), Crocetta del Montello, Terra Ferma, 2010. 
Stanzani / Orsi / Giudici 2001 Anna Stanzani / Oriana Orsi / Corinna Giudici (a cura di), Lo spazio il tempo le opere. Il catalogo del patrimonio culturale, catalogo della mostra (Bologna, Pinacoteca Nazionale, 2001-2002), Cinisello Balsamo, Silvana Editoriale, 2001.

Tamassia 1996 Marilena Tamassia, Il Gabinetto Fotografico della Soprintendenza per i Beni Artistici e Storici di Firenze, in Sauro Lusini (a cura di), Fototeche e archivi fotografici. Prospettive di sviluppo e indagine delle raccolte, atti del convegno (Prato, 1992), Prato, Archivio Fotografico Toscano, 1996, pp. 355-358.

Tamassia 2011 Marilena Tamassia (a cura di), Primi anni di attività del Gabinetto Fotografico 1904-1919, Livorno, Sillabe, 2011.

Tamassia 2012 Marilena Tamassia, Primi anni di attività del Gabinetto Fotografico. Seconda parte 1904-1922, Livorno, Sillabe, 2012.

Tamassia 2014a Marilena Tamassia (a cura di), Attività del Gabinetto Fotografico 19161940, Livorno, Sillabe, 2014.

Tamassia 2014b Marilena Tamassia (a cura di), leri. I musei. Allestimenti storici dei musei fiorentini nelle immagini del Gabinetto Fotografico, catalogo della mostra (Firenze, Galleria degli Uffizi, 2014-2015), Livorno, Sillabe, 2014.

Tamassia 2014c Marilena Tamassia, La documentazione delle sculture di Michelangelo nel Gabinetto Fotografico della Soprintendenza fiorentina, in Maffoli / Bietoletti 2014, pp. 78-89.

Tempesti 1994 Fernando Tempesti, I Brogi al tempo dei Brogi, in "AFT. Rivista di storia e fotografia", n. 20, 1994, pp. 74-77.

Tomassini 2003 Luigi Tomassini, L'Italia nei cataloghi Alinari dell'Ottocento. Gerarchie della rappresentazione del "bel paese" fra cultura e mercato, in Carlo Arturo Quintavalle / Monica Maffioli (a cura di), I fratelli Alinari fotografi a Firenze, 150 anni che illustrano il mondo, 1852-1920, Firenze, Alinari, 2003, pp. 147-215.

\#Bernardy 1904 A. Bernardy, Rearranging the Florentine Galleries. A Work of Great Art Importance, in "Boston Evening Transcript", 16 novembre 1904, p. 16, ritaglio consultato nel Fondo Corrado Ricci, Serie Studi Busta 19, Fascicolo 123, Sotto fascicolo 3, Biblioteca Classense, Ravenna.

\#Brogi 1874 Giacomo Brogi, al sig. L. Merciai, lettera ms., Firenze, 13 agosto 1874. Firenze, ASABA, Filza 1874 f. 13.

\#Brogi 1877 Giacomo Brogi e figlio, Al cav. G. Campani, lettera ms., Firenze, 14 febbraio 1877. Firenze, ASGF, Filza 1877 A f. 31.

\#Brogi 1879 Giacomo Brogi e figlio, All'll.mo Signore Commissario Straordinario delle Gallerie e Musei di Firenze, lettera ms., Firenze, 13 marzo 1879. Firenze, ASGF, Filza 1879 B f. 93.

\#Brogi 1881 Brogi, Al sovrintendente, lettera ms., Firenze, 6 giugno 1881. Firenze, ASGF, Filza 1881 A Galleria degli Uffizi f. 15.

\#Brogi 1883 Brogi Giacomo chiede di riprodurre in fotografia alcuni nostri originali, Inventario Firenze, ASGF, Filza 1883 A Galleria degli Uffizi f. 113.

\#Brogi 1885 Brogi fotografo riproduzioni di opere d'arte, Inventario Firenze, ASGF, Filza 1885 Affari generali f. 26.

\#DRRG 1875 DRRG di Firenze, al Sig. Giacomo Brogi Fotografo di Firenze, lettera ms., Firenze, 16 luglio 1875, Firenze, ASGF, Filza 1875 B f. 91. 
\#DRRG 1879a DRRG di Firenze, Al signor Ministro delle Pubblica Istruzione, lettera ms., Firenze, 14 marzo 1879, Firenze, ASGF, Filza 1879 B f. 93.

\#DRRG 1879b DRRG di Firenze, Al Sig. Giacomo Brogi, lettera ms., Firenze, 20 marzo 1879. Firenze, ASGF, Filza 1879 B f. 93.

\#DRRG 1879c DRRG di Firenze, Al Ministro della Pubblica Istruzione, lettera ms., Firenze, 20 marzo 1879, Firenze, ASGF, Filza 1879 B f. 93.

\#DRRG 1879d DRRG di Firenze, Al sig. curato della Chiesa di Ognissanti, lettera ms., Firenze, 22 marzo 1879, Firenze, ASGF, Filza 1879 B f. 93.

\#Filza 1880 Filza 1880 B f. 127, lettere mss. Firenze, ASGF.

\#Filza 1885 Filza 1885 Direzione f. 34, lettere mss. Firenze, ASGF.

\#Filza 1886 Filza 1886 Affari generali f. 9, lettere mss. Firenze, ASGF.

\#Filza 1889 Filza 1889 E, Affari generali, f. 15, lettere mss. Firenze, ASGF.

\#MPI 1867a MPI, al Signor Direttore delle Gallerie Fiorentine, lettera ms., Firenze, 14 ottobre 1867. Firenze, ASGF, Filza 1867 f. 107.

\#MPI 1867b MPI, Al sig. Direttore delle RR. Gallerie di Firenze, lettera ms., Firenze, 27 luglio 1867. Firenze, ASGF, Filza 1867 A f. 83.

\#MPI 1878 MPI, Al sig. Direttore delle RR. Gallerie di Firenze, lettera ms., Roma, 9 febbraio 1878. Firenze, ASGF, Filza 1878 A f. 31.

\#MPI 1879 MPI, All'incaricato della Direzione delle regie gallerie e musei di Firenze, lettera ms., Firenze, 21 marzo 1879. Firenze, ASGF, Filza 1879 B f. 93.

\#Ricci 1903 Corrado Ricci, Archivio Fotografico, foglio a stampa. Firenze, ASGF, Filza 1903, fascicolo 41.

\#Riproduzioni 1887 Riproduzioni di opere d'arte di questi istituti, eseguite dai fotografi Sig. Giacomo Brogi, Fratelli Alinari, G. Paganori e altri, Inventario, Firenze, ASGF, Filza 1887 Affari generali f. 3.

\#Riproduzioni 1888 Riproduzioni degli oggetti d'arte delle RR. Gallerie e Musei eseguite dai Fotografi Braun Ad., Bruckmann Federigo, Brogi Giacomo, Borgiotti Giuseppe, Fineschi Guido, Giannini Egidio, Richter, Inventario, Firenze, ASGF, Filza 1888 Affari generali f. 2.

\#Riproduzioni fotografiche 1882 Riproduzioni fotografiche di Brogi, Inventario, Firenze, ASGF, Filza 1882 D f. 303.

\#Sovrintendente Bossi Segrez 1881 Sovrintendente Bossi Segrez, Al Ministro della Pubblica Istruzione, lettera ms., Firenze, 7 febbraio 1881, Firenze, ASGF, Filza 1881 A Galleria degli Uffizi f. 15 